\title{
Effects of a low-fat, high-carbohydrate diet on VLDL- triglyceride assembly, production, and clearance
}

\author{
Elizabeth J. Parks, ${ }^{1}$ Ronald M. Krauss, ${ }^{2}$ Mark P. Christiansen, ${ }^{3}$ Richard A. Neese ${ }^{3}$ \\ and Marc K. Hellerstein ${ }^{3,4}$
}

\begin{abstract}
${ }^{1}$ Department of Food Science and Nutrition, University of Minnesota-Twin Cities, St. Paul, Minnesota 55108, USA
${ }^{2}$ Department of Molecular and Nuclear Medicine, Life Sciences Division, Lawrence Berkeley National Laboratory, Berkeley, California 94720, USA

${ }^{3}$ Department of Medicine, University of California-San Francisco, and the Medical Service, San Francisco General Hospital, San Francisco, California 94110, USA

${ }^{4}$ Department of Nutritional Sciences, University of California-Berkeley, Berkeley, California 94720, USA
\end{abstract}

Address correspondence to: Elizabeth J. Parks, Department of Food Science and Nutrition, University of Minnesota-Twin Cities, St. Paul, Minnesota 55108, USA. E-mail: eparks@tc.umn.edu. Or to: Marc K. Hellerstein, Department of Nutritional Sciences, University of California-Berkeley, Berkeley, California 94720-3104, USA. Phone: (510) 642-0646; Fax: (510) 642-0535; E-mail: march@nature.berkeley.edu.

Portions of this work were presented in abstract form at the American Diabetes Association National Meeting, June 1998, in Chicago, Illinois, USA.

Received for publication February 17, 1999, and accepted in revised form August 30, 1999.

Low-fat, high-carbohydrate (LF/HC) diets commonly elevate plasma triglyceride (TG) concentrations, but the kinetic mechanisms responsible for this effect remain uncertain. Subjects with low TG (normolipidemic [NL]) and those with moderately elevated TG (hypertriglyceridemic [HTG]) were studied on both a control and an LF/HC diet. We measured VLDL particle and TG transport rates, plasma nonesterified fatty acid (NEFA) flux, and sources of fatty acids used for the assembly of VLDL-TG. The $\mathrm{LF} / \mathrm{HC}$ diet resulted in a $60 \%$ elevation in TG, a $37 \%$ reduction in VLDL-TG clearance, and an $18 \%$ reduction in whole-body fat oxidation, but no significant change in VLDL-apo B or VLDL-TG secretion rates. Significant elevations in fasting apo B-48 concentrations were observed on the LF/HC in HTG subjects. In both groups, fasting de novo lipogenesis was low regardless of diet. The NEFA pool contributed the great majority of fatty acids to VLDL-TG in NL subjects on both diets, whereas in HTG subjects, the contribution of NEFA was somewhat lower overall and was reduced further in individuals on the LF/HC diet. Between 13\% and 29\% of VLDL-TG fatty acids remained unaccounted for by the sum of de novo lipogenesis and plasma NEFA input in HTG subjects. We conclude that (a) whole-food LF/HC diets reduce VLDL-TG clearance and do not increase VLDL-TG secretion or de novo lipogenesis; (b) sources of fatty acids for assembly of VLDL-TG differ between HTG and NL subjects and are further affected by diet composition; (c) the presence of chylomicron remnants in the fasting state on $\mathrm{LF} / \mathrm{HC}$ diets may contribute to elevated TG levels by competing for VLDL-TG lipolysis and by providing a source of fatty acids for hepatic VLDL-TG synthesis; and (d) the assembly, production, and clearance of elevated plasma VLDL-TG in response to LF/HC diets therefore differ from those for elevated TG on higher-fat diets.

J. Clin. Invest. 104:1087-1096 (1999).

\section{Introduction}

Increases in fasting plasma triglyceride (TG) concentrations are commonly observed during the consumption of low-fat, high-carbohydrate (LF/HC) diets $(1,2)$. Other serum lipid changes include reductions in HDL, LDL, and total cholesterol concentrations. The potential atherogenicity of carbohydrate-induced elevations in TG has been the subject of current debate (1-5). Endogenous hypertriglyceridemia, which is observed in individuals on higher-fat diets, is associated with increased coronary heart disease risk (6), but it is not known whether carbohydrate-induced and endogenous TG elevations share underlying kinetic mechanisms and therefore similar atherogenic risk.

Differences in lipoprotein dynamics between the 2 forms of hypertriglyceridemia might contribute differently to the risk for cardiovascular disease. In the fasting state, most plasma TGs are carried in VLDLs. Overpro- duction of VLDL particles might generate a higher flux of atherogenic particles to LDLs in the plasma or into the artery wall. By contrast, reduced clearance of VLDL-TG does not increase the flux of cholesterol into the plasma. Moreover, nonpharmacologic regimens, such as weight loss and exercise training, may increase TG clearance.

Synthesis of VLDL-TG, and particle assembly and secretion, have been the subjects of much in vitro research (7-10). Much of this work, however, has been performed in HepG2 cells, which exhibit defective TG mobilization $(9,10)$. In humans, evidence has been presented for similar TG metabolism in the endogenous and carbohydrate-induced forms of hypertriglyceridemia. TG secretion rates are elevated in endogenous hypertriglyceridemia (11-15), and some (12, 16-19), but not all $(20,21)$, workers have reported high TG flux rates in subjects fed LF/HC diets. Nestel et al. (22) and Boberg et al. $(23,24)$ suggested, on the basis of indirect evidence, 
that the source of fatty acids supporting elevated TG synthesis on $\mathrm{LF} / \mathrm{HC}$ diets may have been from de novo lipogenesis. If increases in dietary carbohydrate elevate de novo lipogenesis, this increase in fatty acid availability to the liver could drive hepatic TG overproduction.

Most previous studies examining the kinetic mechanisms of carbohydrate-induced hypertriglyceridemia have used either short-term ( $<10$ days) feeding protocols $(12,13,15)$, liquid diets nearly devoid of fat $(19,25,26)$, or diets in which the carbohydrate was primarily in the form of simple sugars $(12,19,27)$. Such high-sugar or liquid-formula diets may be metabolized differently than whole-food, high-fiber, low-fat diets consumed normally. Furthermore, the contribution from de novo lipogenesis to fatty acids in TG had not been quantified reliably, owing to the lack of accurate methods to measure this process in humans (28). Accordingly, the present study was designed to determine the metabolic and kinetic mechanisms underlying carbohydrate-induced elevations in TG in subjects consuming an isoenergetic diet that was composed of whole foods, rich in fiber and restricted in monosaccharides. Normolipidemic subjects and those with mildly elevated fasting TG levels were compared to determine whether these groups differed in their response to the $\mathrm{LF} / \mathrm{HC}$ diet.

\section{Methods}

Human subjects. Volunteers were recruited by advertisement and gave written informed consent before enrolling in the study. The University of California-Berkeley and University of California-San Francisco Committees on Human Research approved the protocols. Subjects were eligible for inclusion in the study if their screening TG concentration was less than $100 \mathrm{mg} / \mathrm{dL}$ (normolipidemic [NL]; $n=6$ ), or was 100-200 mg/dL (hypertriglyceridemic [HTG]; $n=5$ ). We chose to study subjects with moderately elevated TG, on the basis of previous work (E.J. Parks, unpublished data; and ref. 29) showing that carbohydrateinduced elevations in TG tend to be more variable in indi-

Table 1

Composition of experimental $\operatorname{diets}^{\mathrm{A}}$

$\begin{array}{lcc} & \text { Control diet } & \text { LF/HC diet } \\ \text { Energy (kJ/d) } & 11,929 \pm 1,172 & 12,209 \pm 971 \\ \text { Fat } & 110 \pm 10 & 49 \pm 4^{\mathrm{B}} \\ \text { g/d } & 35 \pm 1 & 15 \pm 1^{\mathrm{B}} \\ \text { Percent of energy } & 37 \pm 4 & 18 \pm 1^{\mathrm{B}} \\ \text { SFA (g/d) } & & \\ \text { Carbohydrate } & 354 \pm 39 & 499 \pm 41^{\mathrm{B}} \\ \text { g/d } & 50 \pm 1 & 68 \pm 3^{\mathrm{B}} \\ \text { Percent of energy } & & \\ \text { Simple sugars } & 160 \pm 27 & 220 \pm 35^{\mathrm{B}} \\ \text { g/d } & 45 \pm 5 & 44 \pm 4 \\ \text { Percent of total carbohydrate } & 111 \pm 14 & 121 \pm 13^{\mathrm{B}} \\ \text { Protein } & 16 \pm 1 & 17 \pm 1^{\mathrm{B}} \\ \text { g/d } & 30 \pm 5 & 45 \pm 4^{\mathrm{B}} \\ \text { Percent of energy } & 283 \pm 35 & 150 \pm 22^{\mathrm{B}} \\ \text { Fiber (g/d) } & 2: 1 & 1.7: 1 \\ \text { Cholesterol (g/d) } & & \\ \text { Unsat/sat FA ratio (g/g) } & \\ \quad \text { AData are mean } \pm \text { SD. BSignificant difference between the diets }(P<0.05) \text {. Control } \\ \text { diet, 35\% fat diet; Unsat, unsaturated fatty acid (polyunsaturated plus monoun- } \\ \text { saturated); SFA, saturated fatty acid. }\end{array}$

viduals with higher TG concentrations (>250 mg/dL), whereas those with $\mathrm{TG}$ in this moderate range have a more reproducible response to $\mathrm{LF} / \mathrm{HC}$ diets. TG values represented the average of at least 2 measurements, obtained approximately 2 weeks apart in subjects who had fasted 12 hours, were well hydrated, and had abstained from alcohol for at least 48 hours. All subjects were nonsmoking, healthy males who had normal liver enzyme concentrations and no history of medical disorders (liver disease, kidney disease, diabetes mellitus, etc.). One HTG subject had been on a hypolipidemic medication (niacin) previously; this subject's medication had been discontinued for 9 weeks before entry into the study. NL and HTG subjects did not differ in age ( $31 \pm 7$ and $37 \pm 4$ years, respectively; $P=0.25)$, but were significantly different in body weight (73.1 \pm 6.9 and $87.3 \pm 3.2 \mathrm{~kg}$, respectively; $P=0.008$ ), body mass index (BMI; $22.4 \pm 1.3$ and $27.6 \pm 1.1 \mathrm{~kg} / \mathrm{m}^{2}$, respectively; $P=0.008)$, and percentage of body fat $(16.4 \pm 3.3$ and $26.5 \pm 1.1 \%$, respectively; $P=0.001$ ).

Study design. Subjects consumed 2 isoenergetic diets: a control diet (35\% fat) for 1 week, followed by an LF/HC (15\% fat) diet for 5 weeks. The composition of the 2 diets is shown in Table 1. Foods making up the control diet were chosen to be similar to those routinely consumed by the subjects ad libitum during screening. Ad libitum consumption was determined over a period of 2-6 weeks, by 3 methods: 3-day food records, food-frequency questionnaires, and diet history interviews. Only those subjects whose ad libitum dietary fat intake was $30-35 \%$ were enrolled in the study. Both screening and subject participation in the study were scheduled to avoid public holidays and personal events (birthdays, anniversaries, etc.). The LF/HC diet was designed to replace dietary fat with carbohydrate, while limiting the consumption of monosaccharides and sucrose. As typically occurs when fat intake is reduced using whole foods, the content of other dietary components (e.g., cholesterol, fiber, and saturated fat) also changed (Table 1). Subjects were instructed to limit alcohol intake to 1 drink or less per week (equivalent to $12 \mathrm{oz}$ of beer, $8 \mathrm{oz}$ of wine, or $1 \mathrm{oz}$ of hard liquor). For both diets, all meals, beverages, and snacks were prepared by the staff of the metabolic kitchen at the General Clinical Research Center (GCRC) of San Francisco General Hospital. Each subject reported to the GCRC 3-5 days per week to be interviewed by study staff, get weighed, and consume the main meal of the day. Food to be consumed until the next visit was packed and sent home with the subject for consumption as an outpatient. Subjects were instructed to consume all the study food and not to consume any other food during the 6 -week study period. To ensure that metabolic changes on $\mathrm{LF} / \mathrm{HC}$ diet were not confounded by weight loss, adjustments of dietary energy were made to maintain body weight within $1-2 \mathrm{~kg}$ of baseline. Frequent dietary interviews and inspection of returned food containers were used to assess subject compliance. Body composition was measured by bioelectric impedance analysis (model 1990B; Valhalla Scientific Inc., San Diego, California, USA), using calculations according to manufacturer's equations.

Infusion protocol. Sodium $\left[1-{ }^{13} \mathrm{C}_{1}\right]$ acetate, $[5,5,5-$ $\left.{ }^{2} \mathrm{H}_{3}\right]$ leucine, $\left[1,2,3,4-{ }^{13} \mathrm{C}_{4}\right]$ palmitate, $\left[2-{ }^{13} \mathrm{C}_{1}\right]$ glycerol, and $\left[\mathrm{U}^{13} \mathrm{C}_{6}\right]$ glucose were purchased from Isotec (Miamis- 

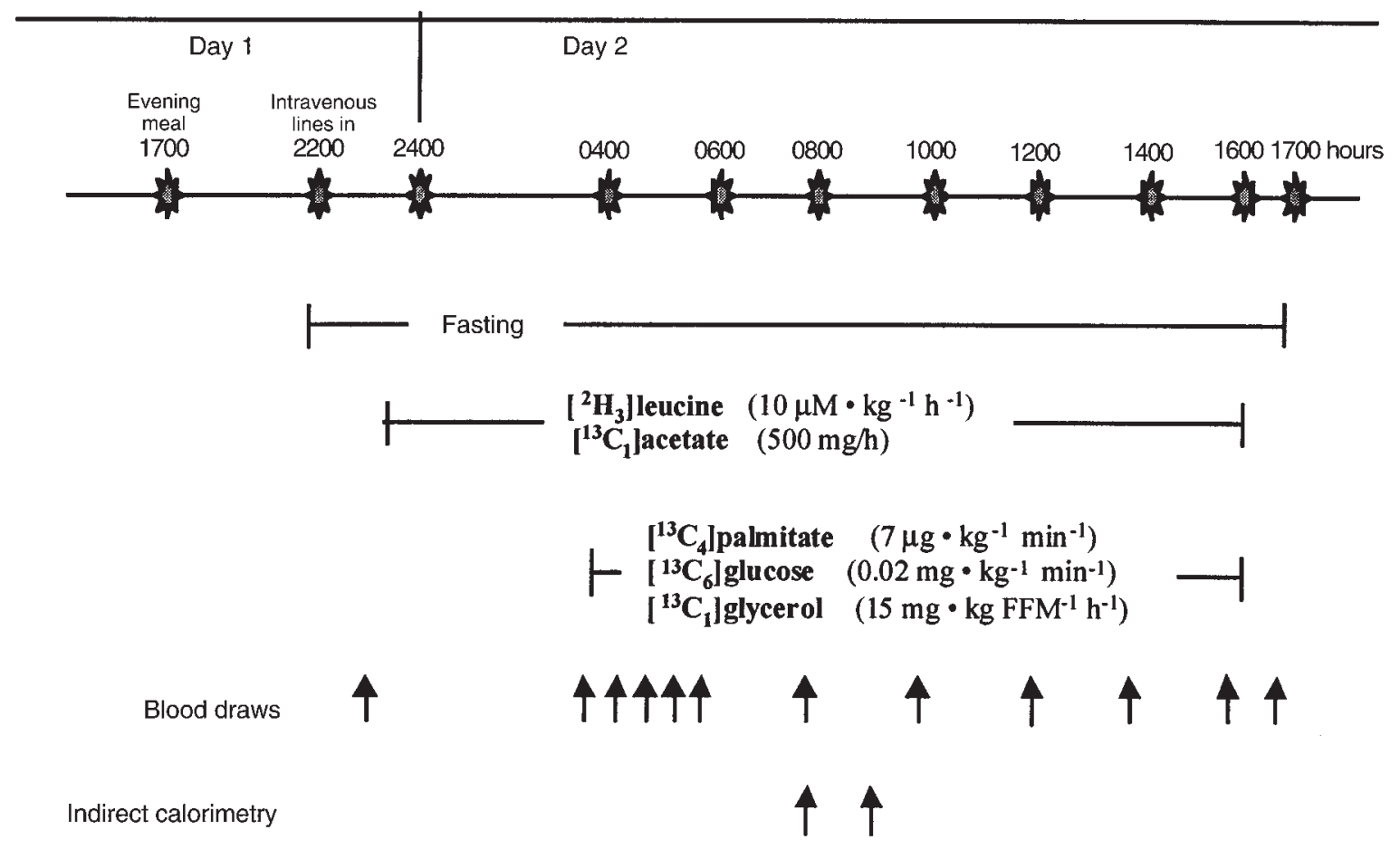

Figure 1

Stable isotope infusion protocol. Subjects were admitted to the GCRC for a 24-hour isotope infusion study; protocol and calculations are described in the text.

burg, Ohio, USA) or Cambridge Isotope Laboratory (Andover, Massachusetts, USA). Isotopic purity was greater than $98 \%$ for all tracers used. The subjects were admitted to the GCRC for 2 stable isotope infusion studies, one administered at the end of each diet phase. The infusion protocol is shown in Figure 1. The evening meal on day 1 (at 1700 hours) reflected the diet composition of the current feeding phase; that is, a $35 \%$ fat meal was fed the night before infusion study no. 1, and a $15 \%$ fat meal was fed the night before infusion study no. 2. After dinner, the subjects remained fasted until the end of the infusion study (1700 hours on day 2). At 2200 hours on day 1 , an intravenous line was placed in the antecubital vein of each arm; one intravenous line was for administration of isotopes, and the other was for drawing blood. At 2400 hours, an infusion was started that contained [1$\left.{ }^{13} \mathrm{C}_{1}\right]$ acetate and $\left[5,5,5-{ }^{2} \mathrm{H}_{3}\right]$ leucine. At 0400 hours on day 2 , a second infusion was started that contained [2${ }^{13} \mathrm{C}_{1}$ ] glycerol, $\left[\mathrm{U}-{ }^{13} \mathrm{C}_{6}\right]$ glucose, and $\left[1,2,3,4-{ }^{13} \mathrm{C}_{4}\right]$ palmitate complexed with human albumin in a molar ratio of 2 fatty acids per albumin (30). Frequent blood samples were drawn between 0400 and 1700 hours on day 2. All blood was drawn into iced Vacutainers containing 1 $\mathrm{mg} / \mathrm{mL}$ EDTA. Plasma was separated immediately by centrifugation $\left(1,500 \mathrm{~g}\right.$ for 20 minutes) at $4^{\circ} \mathrm{C}$, kept on ice, and aliquoted for various analyses. Plasma samples for nonesterified fatty acid (NEFA) analysis were extracted with a 30:70 heptane/isopropanol mixture within 1 hour of the blood draw. An aliquot of plasma was also immediately deproteinized with perchloric acid (2:1, $\mathrm{vol} / \mathrm{vol}$ ) in preparation for isolation of glucose and glycerol by ion exchange chromatography as described previously (31). Subjects rested, watched television, or read during the infusion tests. Non-energy-containing, non- caffeinated drinks (e.g., herbal tea, diet soda) were available upon request. Indirect calorimetry was performed for 30 minutes between 0800 and 0900 hours on day 2. A Deltatrac metabolic cart (Sensor Medix, Yorba Linda, California, USA) in the hooded mode was used.

Analysis of metabolites. Analysis of plasma total, LDL and HDL cholesterol, TG, and apo A-I and B concentrations was performed by staff at the Lipoprotein Laboratory (Lawrence Berkeley National Laboratory, Berkeley, California, USA). Lipids were measured enzymatically on a Ciba-Corning Express Model 550 (Ciba Corning Diagnostics, Oberlin, Ohio, USA), and VLDL cholesterol was calculated by difference. Apolipoproteins were measured by maximal radial immunodiffusion (32). Fasting apo B48 and B-100 concentrations within the $d<1.006 \mathrm{~g} / \mathrm{mL}$ fraction were measured by SDS-PAGE in the laboratory of R. Havel (University of California-San Francisco, San Francisco, California, USA) (33). Concentrations of glucose in plasma samples and in the infusates were measured using a glucose analyzer (Yellow Springs Instrument Co., Yellow Springs, Ohio, USA); plasma insulin concentrations were determined by RIA (Diagnostic Products Corp., Los Angeles, California, USA).

VLDL isolation and subfractionation. Within 24 hours of each infusion study, plasma samples were subjected to ultracentrifugation at $35,000 \mathrm{rpm}$ for 25 minutes in a 50.3 rotor at $15^{\circ} \mathrm{C}\left(1.6 \times 10^{6} \mathrm{~g}\right)$ to separate chylomicrons (34); VLDL $(d<1.006 \mathrm{~g} / \mathrm{mL})$ was then isolated by ultracentrifugation for 20 hours in a 50.3 Beckman rotor $\left(1.3 \times 10^{8}\right.$ $g$ at $12^{\circ} \mathrm{C}$ ). The total VLDL sample from each time point was divided into 2 aliquots. The first aliquot of VLDL was used for isolation of lipoprotein TG by thin-layer chromatography (TLC). After scraping the TG band from the TLC plate, VLDL-TG fatty acids were transesterified to 

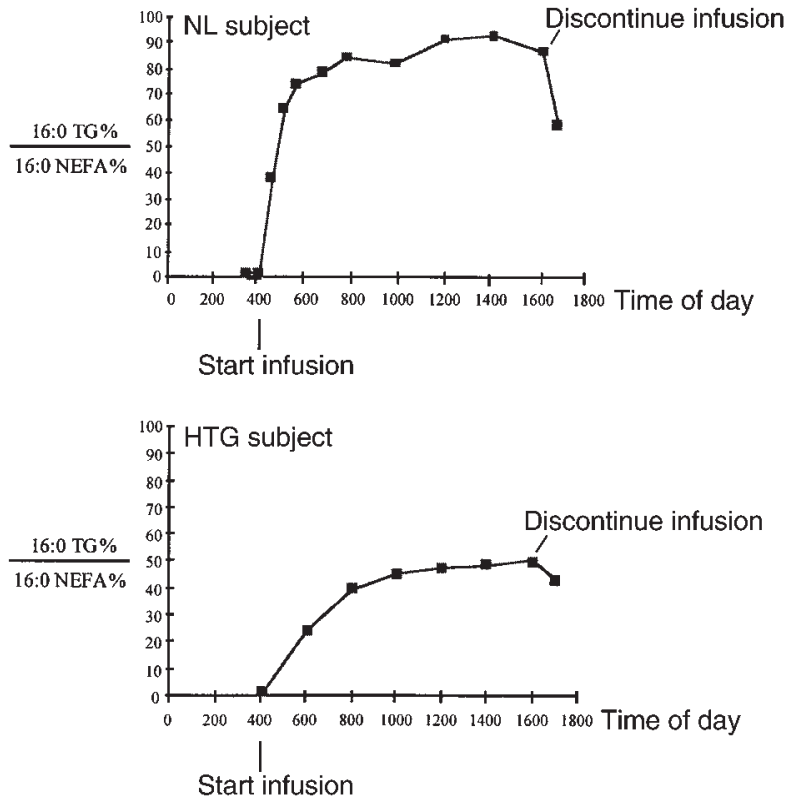

\section{Figure 2}

Percentage of VLDL-TG palmitate derived from the plasma NEFA pool. Data are from 2 representative subjects on the control diet: an NL subject (top) and an HTG subject (bottom). The infusion of $[1,2,3,4$ ${ }^{13} \mathrm{C}_{4}$ ]palmitate (from 0400 to 1600 hours) reached steady state within the plasma NFA pool 1 hour after the start of the infusion.

fatty acid methyl esters, in preparation for gas chromatographic/mass spectrometric analysis (35). The second aliquot of VLDL was subjected to an additional ultracentrifugation step to separate large, buoyant VLDL particles for isolation of apo B. This strategy was used to isolate VLDL that most closely represented particles newly secreted by the liver. The longer VLDL circulates, the more it undergoes remodeling in the plasma compartment (36); the resulting smaller particles have an altered composition and are more prevalent in the plasma of HTG subjects (36). As described previously (37), total VLDL was dialyzed to $d=1.21 \mathrm{~g} / \mathrm{mL}$ with 2 changes of dialysis buffer over a 2day period, layered under a discontinuous gradient of salt solutions of 3 densities $(1.02,1.01$, and $1.0 \mathrm{~g} / \mathrm{mL}$ ), and subjected to nonequilibrium density gradient ultracentrifugation in an SW41 swinging bucket rotor (Beckman Instruments, Palo Alto, California, USA) for 6 hours at $40,000 \mathrm{rpm}$ and $17^{\circ} \mathrm{C}\left(1.8 \times 10^{6} \mathrm{~g}\right)$. The top $1 \mathrm{~cm}^{3}$, denoted large VLDL, was aspirated. Because the sample quantity was limited, large VLDLs were analyzed only for apo B$100\left[5,5,5-{ }^{2} \mathrm{H}_{3}\right]$ leucine enrichment.

Isolation of metabolites and gas chromatographic/mass spectrometric analysis. All gas chromatography/mass spectrometry columns were fused silica and were purchased from J\&W Scientific (Folsom, California, USA). Gas chromatography/mass spectrometry was performed using an HP-5971 instrument (Hewlett-Packard, Palo Alto, California, USA) in the selected-ion monitoring mode. Plasma NEFA, containing $100 \mathrm{nmol}$ pentadecanoic acid (Sigma Chemical Co., St. Louis, Missouri, USA) as an internal standard, were isolated by TLC and transesterified to fatty acid methyl esters. Methyl esters of plasma NEFA and VLDL-TG fatty acids were separat- ed on a DB225 gas chromatography column, and fatty acid composition was measured by flame ionization detection, as described previously (35). The enrichment of $\left[1,2,3,4-{ }^{13} \mathrm{C}_{4}\right]$ palmitate (monitoring $\mathrm{m} / \mathrm{z}: 270,271$, 272 , and 274) was assessed using electron impact ionization and a standard curve. Plasma glucose and free glycerol were isolated by ion exchange chromatography and derivatized with pyridine/acetic anhydride (38). Glucose-pentaacetate was analyzed using a $60-\mathrm{m} \mathrm{DB17}$ column under methane chemical ionization for the molecular ions (monitoring $m / z$ : 331, 332, 333, and 337). Glycerol triacetate was analyzed using a DB225 column under chemical ionization (monitoring $m / z: 159 / 160$ ). Standard curves were used to assess the enrichment of $\left[\mathrm{U}-{ }^{13} \mathrm{C}_{6}\right]$ glucose and $\left[2-{ }^{13} \mathrm{C}_{1}\right]$ glycerol. Large VLDL-apo B was precipitated using the method of Egusa et al. (39). After protein hydrolysis in $6 \mathrm{~N} \mathrm{HCL}$, apo B amino acids were derivatized with MTBSTFA/acetonitrile and separated on a 30-m DB17 gas chromatography column. Positive fragments were measured under electron impact ionization $(m / z: 200$ and 203), and enrichments were calculated using a $\left[5,5,5-{ }^{2} \mathrm{H}_{3}\right]$ leucine standard curve.

Calculations. Calculation of biosynthetic rates was done according to the precursor-product relationship (40). VLDL-TG synthetic rate was calculated in 2 ways from the incorporation of $\left[1,2,3,4-{ }^{13} \mathrm{C}_{4}\right]$ palmitate (40). Fractional replacement (turnover) rates $\left(\mathrm{K}_{\mathrm{s}}\right)$ were calculated by modeling the rise toward plateau enrichment of $[1,2,3,4-$ ${ }^{13} \mathrm{C}_{4}$ ]palmitate into VLDL-TG. The data were fit to the equation $\mathrm{y}=\mathrm{A}_{\infty} \times\left[1-\mathrm{e}^{-\mathrm{K}_{\mathrm{s}}(\mathrm{t}-\mathrm{c})}\right]$, where $\mathrm{y}=\mathrm{VLDL}-\mathrm{TG}$ palmitate enrichment, $\mathrm{A}=$ the plateau or asymptote value of TG palmitate, $\mathrm{t}=$ time in hours, and $\mathrm{c}=$ lag period before isotope incorporation into secreted VLDL-TG. In the same manner, VLDL particle fractional replacement rate $\left(\mathrm{K}_{\mathrm{s}}\right)$ was calculated from the enrichment of $\left[5,5,5-{ }^{2} \mathrm{H}_{3}\right]$ leucine in large VLDL-apo B. Half-lives were calculated by dividing 0.693 by $\mathrm{K}_{\mathrm{s}}(31)$. Plasma volume was estimated as described by Grundy et al. (41) to account for the differences in plasma volume between subjects of different body weight.

The transport rate of total VLDL-TG was determined as follows: VLDL-TG transport rate $(\mu \mathrm{mol} \cdot \mathrm{kg}$ fat-free mass $\left.[\mathrm{FFM}]^{-1} \mathrm{~h}^{-1}\right)=$ VLDL-TG concentration $(\mu \mathrm{mol} / \mathrm{L}) \times$ plasma volume $(\mathrm{L}) \times \mathrm{K}_{\mathrm{s}}\left(\mathrm{h}^{-1}\right) / \mathrm{FFM}(\mathrm{kg})$.

The plasma apo B concentration in large VLDL was determined from the apo B concentration of VLDL after subfractionation. We corrected for dilution due to buffers added during the procedure, but recognize that subfractionation may result in inaccuracies of apo $\mathrm{B}$ pool size measurements. The transport rate of large VLDL-apo B was calculated as follows: Large VLDL-apo B transport rate $\left(\mu \mathrm{mol} \cdot \mathrm{kg}^{-1} \mathrm{~h}^{-1}\right)=$ plasma concentration $(\mu \mathrm{mol} / \mathrm{mL}) \times$ plasma volume $(\mathrm{mL}) \times \mathrm{K}_{\mathrm{s}}\left(\mathrm{h}^{-1}\right) /$ total body weight $(\mathrm{kg})$.

The clearance rate of a metabolite represents the amount of plasma completely cleared of the substance per minute and reflects its efficiency of removal (42). At steady state, clearance was calculated as the ratio of transport or turnover rate to plasma concentration. Thus, to calculate the clearance rates of large VLDL-apo B and VLDL-TG $(\mathrm{mL} / \mathrm{min})$, their transport rates $(\mu \mathrm{mol} / \mathrm{min})$ were divided by their respective plasma concentrations $(\mu \mathrm{mol} / \mathrm{mL})$.

The sources of fatty acids used for the assembly of VLDL-TG were determined as follows. The proportion of 
Table 2

Fasting concentrations of plasma metabolites on the control and LF/HC diets ${ }^{\mathrm{A}}$

\begin{tabular}{|c|c|c|c|c|c|c|}
\hline & \multicolumn{2}{|c|}{ NL subjects } & \multicolumn{2}{|c|}{ HTG subjects } & \multirow{2}{*}{$\begin{array}{c}\text { Comparison of } \\
\text { change scores }^{B} \\
P \text { value }\end{array}$} & \multirow{2}{*}{$\begin{array}{c}\text { Overall } \\
\text { treatment effect } \\
P \text { value }\end{array}$} \\
\hline & Control diet & $\mathrm{LF} / \mathrm{HC}$ diet & Control diet & LF/HC diet & & \\
\hline TG & $61 \pm 7$ & $100 \pm 13$ & $149 \pm 16$ & $228 \pm 24$ & 0.55 & 0.001 \\
\hline Total cholesterol & $131 \pm 9$ & $133 \pm 13$ & $194 \pm 10$ & $174 \pm 7$ & 0.48 & 0.20 \\
\hline LDL cholesterol & $79 \pm 8$ & $67 \pm 9$ & $130 \pm 12$ & $100 \pm 9$ & 0.35 & 0.001 \\
\hline HDL cholesterol & $41 \pm 3$ & $38 \pm 3$ & $34 \pm 3$ & $31 \pm 3$ & 0.68 & 0.03 \\
\hline VLDL cholesterol & $11 \pm 1$ & $27 \pm 8$ & $30 \pm 3$ & $44 \pm 5$ & 0.61 & 0.005 \\
\hline Apo B & $66 \pm 6$ & $67 \pm 10$ & $108 \pm 4$ & $114 \pm 4$ & 0.99 & 0.42 \\
\hline Apo A-I & $98 \pm 9$ & $94 \pm 7$ & $99 \pm 3$ & $96 \pm 6$ & 0.84 & 0.52 \\
\hline Insulin (pmol/L) & $31 \pm 5$ & $34 \pm 9$ & $52 \pm 10$ & $43 \pm 6$ & 0.34 & 0.48 \\
\hline Glucose $(\mathrm{mmol} / \mathrm{L})$ & $4.9 \pm 0.2$ & $5.1 \pm 0.2$ & $5.0 \pm 0.1$ & $5.1 \pm 0.2$ & 0.81 & 0.21 \\
\hline $\mathrm{NEFA}(\mu \mathrm{mol} / \mathrm{L})$ & $424 \pm 112$ & $459 \pm 85$ & $614 \pm 125$ & $617 \pm 85$ & 0.90 & 0.78 \\
\hline
\end{tabular}

${ }^{A}$ Data are mean \pm SEM. All values are $\mathrm{mg} / \mathrm{dL}$ unless otherwise noted. ${ }^{\mathrm{B}}$ By analysis of covariance: comparison of the change scores of the 2 groups, adjusted for baseline values. 'Statistical significance for the overall change score $(n=11)$.

VLDL-TG palmitate derived from circulating NEFA was calculated by dividing the enrichment of $[1,2,3,4-$ ${ }^{13} \mathrm{C}_{4}$ ]palmitate in VLDL-TG at plateau by the steady-state enrichment of the plasma NEFA pool. The infusion of $\left[1,2,3,4-{ }^{13} \mathrm{C}_{4}\right]$ palmitate was between $0400-1600$ hours, and enrichment within plasma NEFA reached steady state within 1 hour after the start of the infusion (data not shown). The NEFA enrichment used was the average of at least 6 time points. In all subjects, VLDL-TG $[1,2,3,4-$ ${ }^{13} \mathrm{C}_{4}$ ] palmitate enrichment reached a plateau by the 9 th hour of label infusion, and the plateau value was determined from an average of at least the final 2 time points taken. (An example of the calculation for the data of a NL subject follows: the plateau of TG palmitate enrichment was $1.51 \%$ and the steady-state enrichment of plasma nonesterified palmitate was $1.55 \%$. Therefore, $97.4 \%$ $(1.51 / 1.55)$ of TG palmitate was derived from the plasma NEFA pool; Figure 2). The proportion of VLDL-TG palmitate derived from hepatic de novo lipogenesis (i.e., made "new" from acetyl-CoA precursors) was calculated by mass isotopomer distribution analysis. VLDL isolated from plasma drawn at 0800-0900 hours was used for this analysis in 6 subjects; de novo lipogenesis was also measured at 8 additional time points $(0200,0400,0600,1000$, $1200,1400,1600$, and 1700 hours) and found not to vary during the course of the infusion. Mass isotopomer distribution analysis allows calculation of the isotopic enrichment of the true biosynthetic precursor from the labeling pattern in a polymer, as has been described in detail previously (43). The fractional contribution of gluconeogenesis to endogenous glucose production was determined from the incorporation of $\left[2-{ }^{13} \mathrm{C}_{1}\right]$ glycerol into plasma glucose, using mass isotopomer distribution analysis to calculate the isotopic enrichment of the triosephosphate precursor pool (43). The rate of endogenous glucose production was determined by the dilution of [U${ }^{13} \mathrm{C}_{6}$ ]glucose (31). The rate of adipose tissue lipolysis was calculated from the dilution of $\left[1,2,3,4-{ }^{13} \mathrm{C}_{4}\right]$ palmitate and $\left[2-{ }^{13} \mathrm{C}_{1}\right]$ glycerol in plasma (31). Indirect calorimetry was used to measure energy expenditure using standard equations to determine net substrate oxidation, nonprotein respiratory quotient, and energy expenditure (44). A fixed protein catabolism was assumed based on the daily protein intake of the prepared diets.

Statistics. Data are mean \pm SEM. Statistical analyses were performed using SPSS statistical software (SPSS Inc., Chicago, Illinois, USA), with $P<0.05$ considered significant. Variables whose values were not normally distributed (e.g., plasma TG concentration) were log-transformed before statistical analysis. The subjects were stratified by baseline TG values into 2 groups (NL: subjects with TG < $100 \mathrm{mg} / \mathrm{dL}$; and HTG: those with TG $=100-200 \mathrm{mg} / \mathrm{dL}$ ). To compare the 2 groups for change in response, analysis of covariance was performed to control for differences in baseline values. Measures of glucose flux were collected in a subset of subjects ( $3 \mathrm{NL}$ and $3 \mathrm{HTG}$ subjects); analysis of

Table 3

Glucose and fatty acid kinetics and substrate oxidation rates ${ }^{\mathrm{A}}$

\begin{tabular}{|c|c|c|c|c|c|c|}
\hline & \multicolumn{2}{|c|}{ NL subjects } & \multicolumn{2}{|c|}{ HTG subjects } & \multirow{2}{*}{$\begin{array}{c}\text { Comparison of } \\
\text { change scores } \\
P \text { value }\end{array}$} & \multirow{2}{*}{$\begin{array}{c}\text { Overall } \\
\text { treatment effect } \\
P \text { value }\end{array}$} \\
\hline & Control diet & $\mathrm{LF} / \mathrm{HC}$ diet & Control diet & LF/HC diet & & \\
\hline $\begin{array}{l}\text { Glucose flux }{ }^{\mathrm{B}} \\
\text { derived from glycogenolysis }{ }^{\mathrm{B}} \\
\text { derived from GNG } \\
\text { GNG (\% contribution to flux) }\end{array}$ & $\begin{array}{c}9.1 \pm 1.0 \\
5.9 \pm 0.4 \\
3.2 \pm 0.8 \\
35.4 \pm 0.1\end{array}$ & $\begin{array}{c}9.9 \pm 0.4 \\
6.5 \pm 0.4 \\
3.4 \pm 0.4 \\
34.6 \pm 0.1\end{array}$ & $\begin{array}{c}7.2 \pm 0.6 \\
3.8 \pm 0.6 \\
3.4 \pm 0.3 \\
46.7 \pm 0.1\end{array}$ & $\begin{array}{c}7.9 \pm 0.1 \\
4.6 \pm 0.4 \\
3.3 \pm 0.5 \\
41.6 \pm 0.1\end{array}$ & $\begin{array}{l}\text { ND } \\
\text { ND } \\
\text { ND } \\
\text { ND }\end{array}$ & $\begin{array}{l}0.13 \\
0.43 \\
0.58 \\
0.64\end{array}$ \\
\hline $\begin{array}{l}\text { Whole-body glucose oxidation } \\
\mu \mathrm{mol} \cdot \mathrm{kg} \text { TBW } \\
\mu \mathrm{min}^{-1} \\
\mu \mathrm{mol} \cdot \mathrm{kg} \mathrm{FFM}{ }^{-1} \mathrm{~min}^{-1} \\
\text { NEFA flux }\left(\mu \mathrm{M} \cdot \mathrm{kg} \mathrm{fat}^{-1} \mathrm{~min}^{-1}\right)\end{array}$ & $\begin{array}{c}6.0 \pm 1.0 \\
7.5 \pm 1.2 \\
29.8 \pm 5.8\end{array}$ & $\begin{array}{c}8.0 \pm 1.4 \\
9.7 \pm 1.7 \\
24.4 \pm 6.0\end{array}$ & $\begin{array}{c}6.3 \pm 1.5 \\
8.7 \pm 2.2 \\
22.9 \pm 2.7\end{array}$ & $\begin{array}{c}7.8 \pm 2.8 \\
10.7 \pm 3.8 \\
21.7 \pm 5.1\end{array}$ & $\begin{array}{l}0.82 \\
0.68 \\
0.74\end{array}$ & $\begin{array}{l}0.09 \\
0.12 \\
0.16\end{array}$ \\
\hline $\begin{array}{l}\text { Whole-body fat oxidation } \\
\mu \mathrm{mol} \cdot \mathrm{kg} \mathrm{TBW}^{-1} \mathrm{~min}^{-1} \\
\mu \mathrm{mol} \cdot \mathrm{kg} \mathrm{FFM}^{-1} \mathrm{~min}^{-1}\end{array}$ & $\begin{array}{l}4.5 \pm 0.4 \\
5.6 \pm 0.5\end{array}$ & $\begin{array}{l}3.9 \pm 0.5 \\
4.8 \pm 0.6\end{array}$ & $\begin{array}{l}4.2 \pm 0.4 \\
5.7 \pm 0.6\end{array}$ & $\begin{array}{l}3.4 \pm 0.8 \\
4.6 \pm 1.1\end{array}$ & $\begin{array}{l}0.53 \\
0.78\end{array}$ & $\begin{array}{l}0.05 \\
0.02\end{array}$ \\
\hline
\end{tabular}

AData are mean \pm SEM; statistics as defined for Table 2. BMeasurements of glucose flux were performed in only 3 NL and 3 HTG subjects; units of glucose flux are $\mu$ mol $\cdot \mathrm{kg} \mathrm{TBW}^{-1} \mathrm{~min}^{-1}$. GNG, gluconeogenesis; ND, analysis of covariance not performed because of small sample size ( 3 subjects from each group). 
covariance was not performed on these data because the sample size was deemed too small. A $P$ value for the overall treatment effect was also calculated from a paired $t$ test of the entire group $(n=11)$.

\section{Results}

Analysis of diets and comparison of baseline values between NL and HTG subjects. The study diets differed in their percentage of energy from fat, as well as in other characteristics (Table 1). In particular, compared with the control diet, the $\mathrm{LF} / \mathrm{HC}$ diet contained $50 \%$ more fiber and $89 \%$ less cholesterol. The percentage distribution of carbohydrate as simple sugars (sucrose, fructose, glucose, lactose, and maltose) remained constant, although the absolute intake of simple sugars increased by $38 \%$ on the LF/HC diet. There was no difference in baseline intake between the NL and HTG subjects with respect to total energy intake or macronutrient composition of the diet (in grams per day or percentage of energy of fat, protein, or carbohydrate). All subjects tolerated the $\mathrm{LF} / \mathrm{HC}$ diet well, aside from a common complaint of feeling overly full. The groups had significantly different baseline values for TG, total cholesterol, LDL cholesterol, VLDL cholesterol, and apo B ( $P$ $<0.05$ ), with the HTG subjects having higher values for all of these variables (Table 2). HTG subjects tended to have lower concentrations of HDL cholesterol $(P=0.09)$

Plasma lipid and lipoprotein concentrations. The lipid responses to dietary fat reduction are shown in Table 2. All subjects experienced significant elevations in fasting TG concentration on the LF/HC diet. Significant reductions in LDL, HDL cholesterol, and elevations in VLDL cholesterol were observed, with no group effect observed for any of these variables. The relative increase in $\mathrm{TG}$ was similar for both groups, when the TG values were logtransformed (12\% for NL and 9\% for HTG). On the control diet, plasma insulin concentration was borderline/significantly higher in the HTG subjects $(52 \pm 10$ $\mathrm{pmol} / \mathrm{L})$ compared with the NL subjects $(31 \pm 5 ; P=$ 0.06 ); no treatment effect was observed for this variable. The fasting concentrations of apo A-I, glucose, and NEFA remained unchanged with $\mathrm{LF} / \mathrm{HC}$ feeding, with

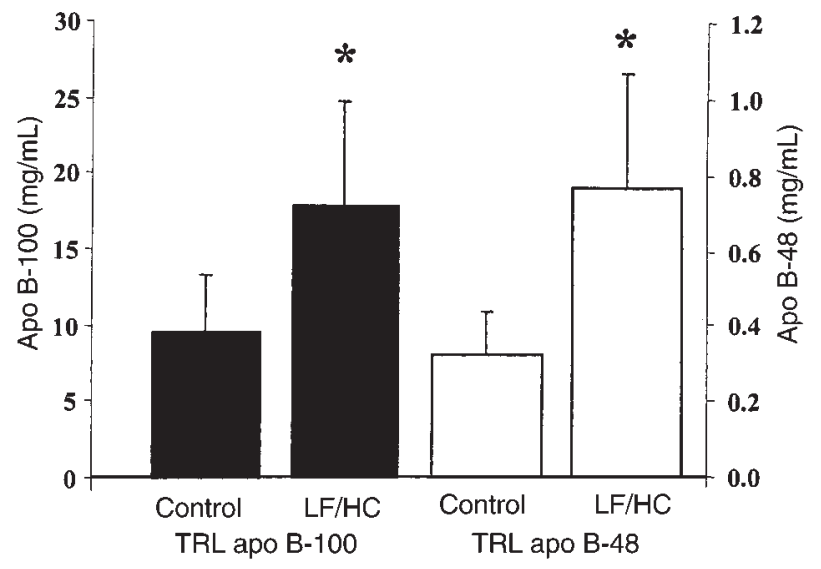

Figure 3

Apolipoprotein (apo) concentrations in the TG-rich lipoprotein (TRL) fraction in HTG subjects. Values are mean \pm SEM. ${ }^{*} P<0.05$, comparison between dietary treatments. no difference in response between the groups. In the HTG group only, fasting concentrations of apo B-100 and B-48 from the TG-rich lipoprotein fraction were measured. LF/HC feeding was associated with significant, 2-fold increases in both the apo B-100 and apo B48 content of the TG-rich lipoprotein fraction (Figure 3 ).

Fuel utilization. Measures of carbohydrate and fat metabolism are presented in Table 3. The energy expenditure of the subjects was not significantly different between the dietary phases (averaging 12,100 kJ/d for all subjects combined). In a subset of subjects (3 from each group), glucose flux data were obtained and showed no effect of diet on glucose production or the sources of endogenous glucose production. The 2 groups did not respond differently to the diets with respect to fatty acid flux or glucose oxidation. For the group as a whole, glucose oxidation was nonsignificantly higher on the LF/HC diet $(P=$ 0.09 , for treatment effect). Whole-body fat oxidation was significantly lower for the entire group after the LF/HC diet ( $18 \%$ reduced; $P=0.02$ for the treatment effect).

Lipoprotein kinetics. The dietary response of the subjects with respect to VLDL particle and TG transport is presented in Table 4. Analysis of covariance revealed that the 2 groups responded differently with respect to large VLDL-apo B transport rate $(P=0.03)$, although neither of the $P$ values for changes in the individual groups was significant $(P<0.13$ and 0.31 for the control and HTG subjects, respectively); this was most likely due to the small sample size and high variability. Use of a different statistical comparison (comparing the means for the groups at a given time point) showed that at baseline, the apo $B$ transport rates were not different between the groups ( 0.11 vs. $0.24 \mu \mathrm{mol} \cdot \mathrm{kg}$ total body weight $[\mathrm{TBW}]^{-1}$ $\mathrm{h}^{-1}$ for the control vs. HTG, respectively; $P=0.31$ ), whereas this difference approached significance after the $\mathrm{LF} / \mathrm{HC} \operatorname{diet}\left(0.13\right.$ vs. $\left.0.32 \mu \mathrm{mol} \cdot \mathrm{kg} \mathrm{TBW}^{-1} \mathrm{~h}^{-1} ; P=0.08\right)$. No group differences were observed for any of the other variables presented in Table 4. For all subjects combined, the mass of large VLDL-apo B was significantly increased on the LF/HC diet (94\% and 51\% for the controls and HTG, respectively); the fractional replacement rate was nonsignificantly slower $(P=0.09)$, but clearance rate did not change. The concentration of TG in the large VLDL fraction was significantly increased in both groups on the LF/HC diet: $62 \%$ in the control and $75 \%$ in the HTG subjects (treatment effect for the entire group: $P=0.04$ ). VLDL-TG transport rate was not different between the dietary phases $(P=0.58)$. By contrast, VLDL-TG fractional replacement rate $\left(\mathrm{K}_{\mathrm{s}}\right)$ was reduced significantly on the $\mathrm{LF} / \mathrm{HC} \operatorname{diet}(P=0.03)$. This slower fractional replacement rate was accompanied by a $37 \%$ reduction in the clearance rate of VLDL-TG (overall $P=0.05$ ), with no significant difference in response between the groups.

Assembly of VLDL-TG: sources of fatty acids. The percentage of VLDL-TG derived from the plasma NEFA pool was determined from the relative enrichments of this pool during infusion of $\left[1,2,3,4-{ }^{13} \mathrm{C}_{4}\right]$ palmitic acid. Representative data from 2 subjects are shown in Figure 2. As demonstrated by the NL subject's data, more than $90 \%$ of palmitic acid found in VLDL-TG derived from the plasma NEFA pool at the end of the 12-hour infusion of label. By contrast, data from the HTG subject 
reveal that at steady state, about $72 \%$ of TG fatty acids were derived from NEFA. The contribution from de novo lipogenesis to palmitate in VLDL-TG was also measured. The summary data (Figure 4) show that de novo lipogenesis did not make a substantial contribution of fatty acids $(<5 \%)$ to fasting TG on either diet, in either group. For NL subjects on both diets, plasma NEFA provided the primary source of palmitate in VLDL-TG (94\% and 92\% for the control and LF/HC, respectively). Compared with the NL subjects, the contribution of NEFA was significantly lower in the HTG subjects on both diets (84\% and 67\% from NEFA on the control and LF/HC diets, respectively). The total percentage of VLDL-TG accounted for by the sum from NEFA and de novo lipogenesis was similar in the NL subjects, regardless of diet (97\%). In the HTG subjects, the total accounted for on the control diet was $87 \%$ (84\% from NEFA, and 3\% from de novo lipogenesis), which was significantly less than that of the NL group $(P=$ 0.02). This difference became greater after the LF/HC diet, when the total accounted for by NEFA plus de novo lipogenesis dropped to $71 \%$ in the HTG (compared with $97 \%$ in NL subjects; $P=0.004$ between groups). Within the HTG group alone, LF/HC feeding significantly reduced the percentage of VLDL-TG palmitate accounted for by fatty acid inputs $(87 \%$ vs. $71 \%$ on the control and $\mathrm{LF} / \mathrm{HC}$ diets, respectively; $P=0.04)$. A significant negative correlation was observed between the percentage of VLDL-TG palmitate derived from the plasma NEFA pool and the body fat percentage in subjects consuming a $35 \%$ fat diet $(r=-0.70, P<0.01$; data not shown). This relationship was similar, although not statistically significant $(r=-0.55, P=0.07)$, after consumption of the $\mathrm{LF} / \mathrm{HC}$ diet.

\section{Discussion}

Fasting TG concentrations increased by $60 \%$ in subjects consuming the solid-food, high-fiber, LF/HC diet. These results underscore the ability of $\mathrm{LF} / \mathrm{HC}$ diets to elevate fasting plasma TG even when the diet is composed of whole foods and is low in simple sugars. Increased secretion of TG was not the primary kinetic mechanism responsible for this carbohydrate-induced TG elevation, however; reduced TG clearance from the plasma was the major metabolic mechanism involved. This conclusion reveals a difference in the

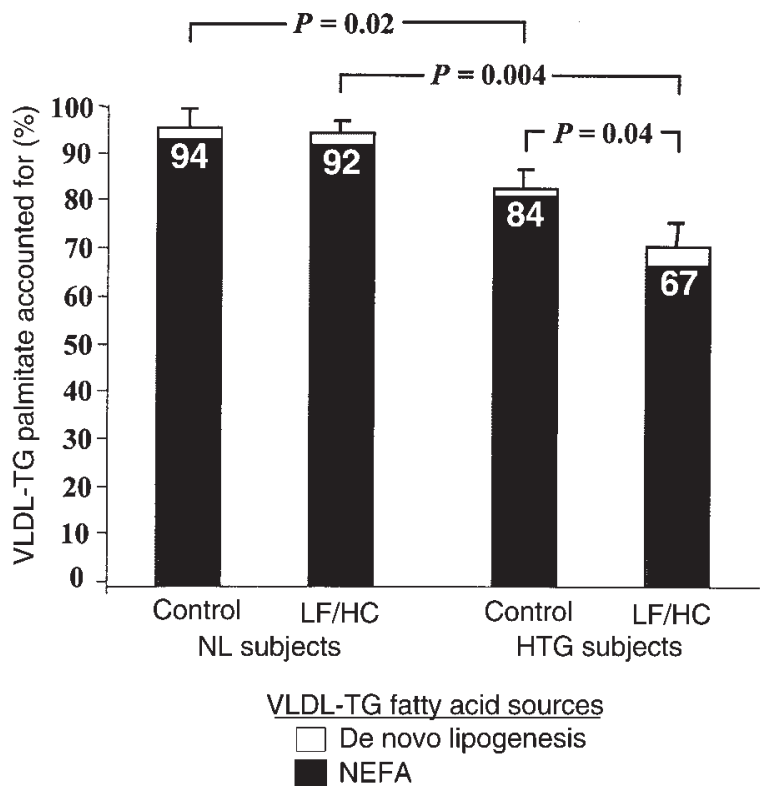

Figure 4

Sources of VLDL-TG palmitic acid. Values are mean \pm SEM. The numbers on the bars represent the percentages of VLDL-TG palmitate derived from the NEFA pool alone (darker portion of the bar).

underlying mechanism from hypertriglyceridemia observed on higher-fat diets. Although lipoprotein kinetic studies are frequently limited in their ability to discern between primary effects on overproduction or reduced clearance of TG, the latter mechanism is supported by several independent measurements. First, the transport rate of VLDL-TG (as assessed by the kinetics of TG palmitate) was not increased on the LF/HC diet. Second, the half-life of VLDL-TG was prolonged, and the clearance of VLDL-TG was significantly reduced. Third, fasting apo B-48 concentrations were elevated. Finally, de novo lipogenesis was not increased.

Previous studies comparing TG secretion in NL individuals with that in those with endogenous hypertriglyceridemia consuming high-fat diets have reported significantly higher rates (11-15). In the present study, the TG secretion rate of HTG subjects was 2.7-fold higher than that of the NL subjects on the higher-fat diet. In contrast, carbohydrate-induced elevations in TG were explained by reduced clearance of VLDL-TG rather than increased pro-

Table 4

Kinetics of VLDL-apo B and VLDL-TGA

\begin{tabular}{|c|c|c|c|c|c|c|}
\hline & \multicolumn{2}{|c|}{ NL subjects } & \multicolumn{2}{|c|}{ HTG subjects } & \multirow{2}{*}{$\begin{array}{c}\text { Comparison of } \\
\text { change scores } \\
\qquad \text { value }\end{array}$} & \multirow{2}{*}{$\begin{array}{c}\text { Overall } \\
\text { treatment effect } \\
P \text { value }\end{array}$} \\
\hline & Control diet & LF/HC diet & Control diet & LF/HC diet & & \\
\hline Concentration $(\mu \mathrm{g} / \mathrm{mL})^{\mathrm{B}}$ & $3.4 \pm 1.1$ & $6.6 \pm 1.6$ & $12.8 \pm 3.8$ & $19.3 \pm 5.2$ & 0.86 & 0.03 \\
\hline Transport rate $\left(\mu \mathrm{mol} \cdot \mathrm{kg} \mathrm{TBW}^{-1} \mathrm{~h}^{-1}\right)^{\mathrm{B}}$ & $0.11 \pm 0.05$ & $0.13 \pm 0.04$ & $0.24 \pm 0.05$ & $0.32 \pm 0.08$ & 0.03 & - \\
\hline$K_{s}\left(h^{-1}\right)^{B}$ & $0.36 \pm 0.05$ & $0.25 \pm 0.04$ & $0.25 \pm 0.05$ & $0.22 \pm 0.06$ & 0.74 & 0.09 \\
\hline Clearance rate $(\mathrm{mL} / \mathrm{min})^{\mathrm{B}}$ & $17.5 \pm 4.4$ & $12.4 \pm 2.2$ & $16.7 \pm 4.4$ & $15.1 \pm 5.3$ & 0.64 & 0.32 \\
\hline \multicolumn{7}{|l|}{ VLDL-TG } \\
\hline Concentration $(\mathrm{mg} / \mathrm{dL})^{\mathrm{B}}$ & $5.0 \pm 1.6$ & $8.1 \pm 1.9$ & $10.2 \pm 2.0$ & $17.8 \pm 5.9$ & 0.29 & 0.04 \\
\hline Transport rate $\left(\mu \mathrm{mol} \cdot \mathrm{kg} \mathrm{FFM}^{-1} \mathrm{~h}^{-1}\right)$ & $10.3 \pm 1.5$ & $12.5 \pm 1.5$ & $27.9 \pm 10.8$ & $18.9 \pm 4.2$ & 0.26 & 0.58 \\
\hline $\mathrm{K}_{\mathrm{s}}\left(\mathrm{h}^{-1}\right)$ & $0.44 \pm 0.04$ & $0.32 \pm 0.06$ & $0.37 \pm 0.08$ & $0.19 \pm 0.05$ & 0.41 & 0.03 \\
\hline Clearance rate $(\mathrm{mL} / \mathrm{min})$ & $23 \pm 3$ & $19 \pm 3$ & $29 \pm 10$ & $12 \pm 4$ & 0.78 & 0.05 \\
\hline
\end{tabular}

AData are mean \pm SEM; statistics as defined for Table 2. ${ }^{B}$ Signifies measurement made on the large VLDL fraction. 
duction. The reduction in TG clearance during $\mathrm{LF} / \mathrm{HC}$ feeding observed here differs from some previous reports of elevated TG production in carbohydrate-induced hypertriglyceridemia $(12,19,27)$. However, we fed a whole-food, high-fiber diet, whereas those previous LF/HC research diets were of liquid form and/or high in mono- and disaccharides $(12,19,27)$. Hudgins et al. (45) have recently shown that the form of carbohydrate (simple sugar vs. starch) markedly influences fatty acid synthesis (de novo lipogenesis; see later here). The form of carbohydrate might also influence TG production. Reduced VLDL-TG clearance may be due to competition between VLDL and chylomicron remnants for TG hydrolysis in the plasma compartment, as fasting apo B-48 concentrations were elevated in the HTG subjects. Other possible causes of reduced VLDL-TG clearance can be proposed (46-48). The mechanisms responsible for reduced VLDL-TG clearance on $\mathrm{LF} / \mathrm{HC}$ diets need further study.

A second goal of this work was to determine whether dietary fat reduction altered the assembly of VLDL-TG, in particular the sources of fatty acids used for TG synthesis. In the NL subjects, NEFA provided more than $93 \%$ of the fatty acids secreted in VLDL-TG by the liver, and the contribution of this source was unchanged by dietary fat reduction. The synthesis of fatty acids de novo contributed less than $5 \%$ of VLDL-TG palmitate, even on the LF/HC diet, excluding hepatic de novo lipogenesis as a basis for increased TG secretion (24). Compared with the NL group, HTG subjects consuming the control diet had significantly lower percentages of TG palmitate derived from plasma NEFA (84\%), with newly synthesized fatty acids accounting for less than 3\% of VLDL-TG palmitate. Thus, $13 \%$ of fatty acid precursors for TG synthesis were derived from neither NEFA nor de novo lipogenesis. The percentage of TG precursors not accounted for by either isotopic labeling route became even greater when HTG subjects consumed the LF/HC $\operatorname{diet}(29 \%$ of VLDL-TG palmitate remaining unaccounted for). Several years ago, Barter and Nestel reported that a substantial percentage of VLDL fatty acids were not derived from NEFA in obese individuals (49). Havel et al. (50) and Boberg et al. (24) also showed that for some HTG subjects, splanchnic NEFA uptake did not explain higher TG secretion rates and that other sources must have provided the precursors for TG synthesis. Furthermore, elevated BMI correlates with lower contributions of plasma NEFA to VLDL-lipid (49). A similar inverse relationship between body fat percentage and plasma NEFA contribution to TG synthesis was observed in the present study on the control diet.

One source of unlabeled TG fatty acids in HTG subjects could be splanchnic lipid, from hepatic or visceral sources (20), as peripheral blood NEFA enrichments are used in the calculation (Figure 2). Obese subjects have increased liver TG content $(51,52)$. Havel et al. (50) observed that about two thirds of the NEFA taken up by the HTG liver escaped oxidation and were apparently stored, with only one third of the NEFA being secreted as TG. This process could lead to the slow turnover of hepatic TG droplets in HTG subjects. In the present study, a contribution from unlabeled hepatic lipid stores to TG synthesis may be less likely, because the subjects had been fasted for 24 hours by the end of the infusion test, which should substantially reduce hepatic lipid stores. Visceral adipose stores are also a potential source of fatty acids for TG assembly. This adipose depot is metabolically active $(53,54)$ and is an important predictor of abnormal plasma lipoprotein concentrations (55). Furthermore, individuals with upper body obesity have elevated VLDL-TG production rates $(41,56,57)$. Visceral fatty acid sources could have contributed to the unlabeled VLDL-TG observed in the HTG subjects on the control diet (Figure 4), but an increase in their contribution on the LF/HC diet is less likely, as we and others (e.g., 58) found no change in body composition on the LF/HC diet.

Finally, an interesting potential source of unlabeled lipid for TG assembly on the LF/HC diet is chylomicron remnants cleared to the liver. In rats, chylomicron remnants contain approximately $50 \%$ of their original TG when cleared to the liver $(59,60)$. Very little is known about this process in humans. The 2-fold elevation in fasting apo B-48 concentrations observed in the HTG subjects after the $\mathrm{LF} / \mathrm{HC}$ diet suggests that chylomicron remnants might provide a significant source of VLDL-TG fatty acids in the fasting state. The increased presence of B-48-containing TG-rich lipoproteins was surprising, given that the blood draw for this measurement occurred after the subjects had fasted 15 hours and that the last meal they consumed contained very little fat ( $15 \%$ of energy). However, Karpe et al. (61) have shown that the chylomicron remnant pool contains lipoprotein populations that are heterogeneous. One population may have a very short half-life ( $<15$ minutes), whereas others may circulate for much longer periods. The elevation in fasting apo B-48 associated with LF/HC feeding could have resulted from alterations in the intestinal secretion pattern of chylomicrons, such that more particles were secreted in the fasting state or that a subpopulation of the chylomicrons secreted postprandially circulated for a longer time. This result requires further investigation.

Another somewhat surprising result was the role of de novo lipogenesis in the response to the $\mathrm{LF} / \mathrm{HC}$ diet. In the present study, the proportion of fatty acids synthesized de novo was extremely low, contrary to the results of our previous collaborative study with Hudgins et al. (27). Because the 2 investigations were similar with respect to duration of the high-carbohydrate diet, baseline TG concentrations of the subjects, the magnitude of TG responses to the diets, and the protocols used for infusion of labeled acetate, the most likely explanation for the difference between the 2 studies is the composition of the LF/HC diets administered. The liquid diet, high in simple sugars, that was used previously (27) might be more lipogenic than the present whole-food, $\mathrm{LF} / \mathrm{HC}$ diet. In a subsequent study, Hudgins et al. (45) reported that consumption of longer-chain dietary carbohydrates, compared with shortchain glucose polymers and simple sugars, reduced the lipogenic response to low-fat feeding (45).

Subjects in this study received only 1 week of the prepared high-fat diet. A crossover design, with each diet fed for 5 weeks, would have been ideal but would have made subject compliance more difficult. Moreover, we took a number of precautions, including the use of 3 methods to assess baseline food intake, avoidance of screening over 
holidays or vacations, and the use of several screening visits for assessment of the subjects' background diets. With the baseline intake so well documented, we therefore consider it likely that the baseline research diet, containing $35 \%$ fat, was similar to the subjects' background diets.

In conclusion, carbohydrate-induced TG elevations occurred in both NL and HTG subjects switched from a higher-fat diet (35\%) to a $15 \%$ fat, whole-food diet. The elevations in fasting TG concentration can be explained kinetically by reduced clearance of VLDL-TG concurrent with elevations in fasting chylomicron remnant concentrations in the HTG subjects. Production of plasma VLDL-TG was higher in HTG subjects compared with NL subjects on the higher-fat diet, but was not increased by the LF/HC diet, and fasting de novo lipogenesis remained low ( $<5 \%$ of VLDL-TG fatty acids, even on the $\mathrm{LF} / \mathrm{HC}$ diet). The lack of stimulation of de novo lipogenesis with a whole-food, complex carbohydrate diet is in contrast to observations with simple sugars in the diet (45). This disparity has relevance to the current increase in consumption of commercially available low-fat foods, in that some $\mathrm{LF} / \mathrm{HC}$ diets may be more lipogenic than others. In the present study, sources other than NEFA and de novo lipogenesis contributed significantly to VLDL-TG assembly in HTG subjects, and these sources contributed even more when HTG subjects consumed the $\mathrm{LF} / \mathrm{HC}$ diet. The assembly, production, and clearance of elevated plasma VLDL-TG in response to the LF/HC diet differed from that associated with the elevated TG on the higher-fat diet. Caution should be used in extrapolating our findings to individuals with frank hypertriglyceridemia (TG > $300 \mathrm{mg} / \mathrm{dL}$ ), because our subjects were recruited to have moderate elevations in baseline TG concentration $(100-200 \mathrm{mg} / \mathrm{dL})$. The results presented here, however, have potential public health significance, as this moderate degree of hypertriglyceridemia is highly prevalent in the population (62).

\section{Acknowledgments}

This work was done during tenure of a Postdoctoral Research Fellowship (to E.J. Parks) from the American Heart Association, California Affiliate. The project was supported, in part, by funds from the Nora Eccles Treadwell Foundation, the International Life Sciences Institute-North America, and Kellogg Inc., and by GCRC grant 5-M01-RR00083-37 from the Division of Research Resources, National Institutes of Health (NIH). Additional support was provided by a grant from the National Dairy Promotion and Research Board (to R.M. Krauss), administered in cooperation with the National Dairy Council, and by NIH Program Grant HL-18574 from the National Heart, Lung, and Blood Institute through the US Department of Energy under contract DE-AC0376SF00098. The authors express their appreciation to Leila Kotite at University of California-San Francisco, Doris Dare, Kristie Frazier, and the nurses of the GCRC staff at San Francisco General Hospital for excellent subject care and data collection; to Mark Hudes for statistical advice; and to Katherine Haddock, Ken Abe, Eva Hughes, and the staff of Lipoprotein Analysis Laboratory of Lawrence Berkeley National Laboratory for their skilled technical assistance.
1. Connor, W.E., and Connor, S.L. 1997. Should low-fat, high-carbohydrate diets be recommended for everyone? N. Engl. J. Med. 337:562-563.

2. Katan, M.B., Grundy, S.M., and Willett, W.C. 1997. Beyond low-fat diets. N. Engl. J. Med. 337:563-566.

3. Ornish, D. 1998. Low-fat diets [letter]. N. Engl. J. Med. 338:128.

4. Rudel, L.L. 1998. Low-fat diets [letter]. N. Engl. J. Med. 338:127.

5. Manning, A. Study: lowfat diets can go too far. USA Today. November 12, 1997. p. 1D.

6. Austin, M.A., Holkanson, J.E., and Edwards, K.L. 1998. Hypertriglyceridemia as a cardiovascular risk factor. Am. J. Cardiol. 81:7B-12B.

7. Cianflone, K., Dahan, S., Monge, J.C., and Sniderman, A.J. 1992. Pathogenesis of carbohydrate-induced hypertriglyceridemia using HepG2 cells as a model system. Arterioscler. Thromb. 12:271-277.

8. Boren, J., Wettesten, M., Rustaeus, S., Andersson, M., and Olofsson, S.-O. 1993. The assembly and secretion of apoB-100 containing lipoproteins. Biochem. Soc. Trans. 21:487-493.

9. Wu, X., Shang, A., Jiang, H., and Ginsberg, H. 1996. Low rates of apoB secretion from HepG2 cells result from reduced delivery of newly synthesized triglyceride to a "secretion-coupled" pool. J. Lipid. Res. 37:1198-1206.

10. Gibbons, G.F., and Wiggins, D. 1995. Intracellular triacylglycerol lipase: its role in the assembly of hepatic very-low-density-lipoprotein (VLDL). In Advances in enzyme regulation. Volume 35. Elsevier Science Ltd. Oxford, United Kingdom. 179-198.

11. Reaven, G.M., Lerner, R.L., Stern, M.P., and Farquhar, J.W. 1967. Role of insulin in endogenous hypertriglyceridemia. J. Clin. Invest. 46:1756-1767.

12. Ginsberg, H., Olefsky, J.M., Kimmerling, G., Crapo, P., and Reaven, G.M. 1976. Induction of hypertriglyceridemia by a low-fat diet. J. Clin. Endocrinol. Metab. 42:729-735.

13. Reaven, G.M., and Bernstein, R.M. 1978. Effect of obesity on the relationship between very low density lipoprotein production rate and plasma triglyceride concentration in normal and hypertriglyceridemic subjects. Metabolism. 27:1047-1054.

14. Melish, J., Le, N.A., Ginsberg, H., Brown, W.U., and Steinberg, D. 1980. Dissociation of the rates of production of apoprotein B and TG of verylow density lipoproteins during high-carbohydrate feeding in man. Am. J. Physiol. 239:E354-E362.

15. Huff, M.W., and Nestel, P.J. 1982. Metabolism of apolipoproteins CII, CIII1, CIII2 and VLDL-B in human subjects consuming high carbohydrate diets. Metabolism. 31:493-498.

16. Knittle, J.L., and Ahrens, E.K.J. 1964. Carbohydrate metabolism in two forms of hyperglyceridemia. J. Clin. Invest. 43:485-495.

17. Kuo, P.T., and Bassett, D.R. 1965. Dietary sugar in the production of hyperglyceridemia. Ann. Intern. Med. 62:1199-1212.

18. Reaven, G.M., Hill, D.B., Gross, R.C., and Farquhar, J.W. 1965. Kinetics of triglyceride turnover of very low density lipoproteins in human plasma. J. Clin. Invest. 44:1826-1833.

19. Farquhar, J.W., Frank, A., Gross, R.C., and Reaven, G.M. 1966. Glucose, insulin, and triglyceride responses to high and low carbohydrate diets in man. J. Clin. Invest. 45:1648-1656.

20. Barter, P.J., Nestel, P.J., and Carroll, K.F. 1972. Precursors of plasma triglyceride fatty acid in humans. Effects of glucose consumption, clofibrate administration, and alcoholic fatty liver. Metabolism. 21:117-124.

21. Abbott, W.G., et al. 1990. Effect of a high-carbohydrate, low-saturated-fat diet on apolipoprotein B and triglyceride metabolism in Pima Indians. J. Clin. Invest. 86:642-650.

22. Nestel, P.J., Carroll, K.F., and Havenstein, N. 1970. Plasma triglyceride response to carbohydrates, fats and calorie intake. Metabolism. 19:1-18.

23. Boberg, J., Carlson, L.A., Freyschuss, U., Lassers, B.W., and Wahlqvist, M.L. 1972. Splanchnic secretion rates of plasma triglycerides and total and splanchnic turnover of plasma free fatty acids in men with normo- and hypertriglyceridaemia. Eur. J. Clin. Invest. 2:454-466.

24. Boberg, J., Carlson, L.A., and Freyschuss, U. 1972. Determination of splanchnic secretion rate of plasma triglycerides and of total and splanchnic turnover of plasma free fatty acids in man. Eur. J. Clin. Invest. 2:123-132.

25. Schonfeld, G. 1970. Changes in the composition of very low density lipoprotein during carbohydrate induction in man. J. Lab. Clin. Med. 75:206-211.

26. Ginsberg, H.N., Le, N.A., Melish, J., Steinberg, D., and Brown, W.V. 1981. Effect of a high carbohydrate diet on apoprotein-B catabolism in man. Metabolism. 30:347-353.

27. Hudgins, L.C., et al. 1996. Human fatty acid synthesis is stimulated by a eucaloric low fat, high carbohydrate diet. J. Clin. Invest. 97:1-11.

28. Hellerstein, M.K., et al. 1991. Measurement of de novo hepatic lipogenesis in humans using stable isotopes. J. Clin. Invest. 87:1841-1852.

29. Glueck, C.J., Levy, R.I., and Fredrickson, D.S. 1969. Immunoreactive insulin, glucose intolerance, and carbohydrate inducibility in types II, III, IV and V hyperlipoproteinemia. Diabetes. 18:739-747.

30. Christie, W.W. 1982. Lipid analysis. Pergamon Press. New York, NY. 52-53.

31. Wolfe, R.R. 1992. Radioactive and stable isotope tracers in biomedicine. John Wiley \& Sons. New York, NY. 424-426. 
32. Cheung, M.C., and Albers, J.J. 1977. The measurement of apolipoprotein AI and A-II levels in men and women by immunoassay. J. Clin. Invest. 60:43-50. 33. Bergeron, N., Kotite, L., and Havel, R.J. 1996. Simultaneous quantification of apolipoproteins B-100, B-48, and E separated by SDS-PAGE. Methods Enzymol. 268:82-94.

34. Hatch, F.T. 1968. Practical methods for plasma lipoprotein analysis. Adv. Lipid Res. 6:1-68.

35. Parks, E.J., et al. 1998. Reduced susceptibility of LDL from patients participating in an intensive atherosclerosis treatment program. Am. J. Clin. Nutr. 68:778-785.

36. Marzetta, C.A., Foster, D.M., and Brunzell, J.D. 1990. Conversion of plasma VLDL and IDL precursors into various LDL subpopulations using density gradient ultracentrifugation. J. Lipid. Res. 31:975-984.

37. Musliner, T.A., Giotas, C., and Krauss, R.M. 1986. Presence of multiple subpopulations of lipoproteins of intermediate density in normal subjects. Arteriosclerosis. 6:79-87.

38. Siler, S.Q., Neese, R.A., Parks, E.J., and Hellerstein, M.K. 1998. VLDLtriglyceride production after alcohol ingestion, studied using [2${ }^{13} \mathrm{C} 1$ ]glycerol. J. Lipid Res. 39:2319-2328.

39. Egusa, G., Brady, D., Grundy, S.M., and Howard, B.V. 1983. Isopropanol precipitation method for the determination of apoB specific activity and plasma concentrations during metabolic studies of VLDL and LDL apoB. J. Lipid Res. 24:1261-1267.

40. Zilversmit, D.B. 1960. The design and analysis of isotope experiments. Am. J. Med. 29:832-848.

41. Grundy, S.M., Mok, H.Y.I., Zech, L., Steinberg, D., and Berman, M. 1979. Transport of very low density lipoprotein triglycerides in varying degrees of obesity and hypertriglyceridemia. J. Lipid. Res. 63:1274-1283.

42. Ryan, W.G., and Schwartz, T.B. 1965. Dynamics of plasma triglyceride turnover in man. Metabolism. 14:1243-1254.

43. Hellerstein, M.K. 1995. Methods for measurement of fatty acid and cholesterol metabolism. Curr. Opin. Lipidol. 6:172-181.

44. Jequier, E., Acheson, K., and Schutz, Y. 1987. Assessment of energy expenditure and fuel utilization in man. Annu. Rev. Nutr. 7:187-208.

45. Hudgins, L.C., Siedman, C.E., Diakun, J., and Hirsch, J. 1998. Human fatty acid synthesis is reduced after the substitution of dietary starch for sugar. Am. J. Clin. Nutr. 67:631-639.

46. Lithell, H., Jacobs, I., Vessby, B., Hellsing, K., and Karlsson, J. 1982. Decrease of lipoprotein lipase activity in skeletal muscle in man during a short-term carbohydrate-rich dietary regimen; with special reference to HDL-cholesterol apolipoprotein and insulin concentrations. Metabolism. 31:994-998.

47. Farese, R.V., Jr., Yost, T.J., and Eckel, R.H. 1991. Tissue-specific regulation of lipoprotein lipase activity by insulin/glucose in normal-weight humans. Metabolism. 40:214-216.
48. Dreon, D.M., Fernstrom, H.A., Miller, B., and Krauss, R.M. 1994. Lowdensity lipoprotein subclass pattern and lipoprotein response to a reduced-fat diet in men. FASEB J. 8:121-126.

49. Barter, P.J., and Nestel, P.J. 1973. Precursors of plasma triglyceride fatty acids in obesity. Metabolism. 22:779-785.

50. Havel, R.J., Kane, J.P., Segel, E.O., and Basso, L.V. 1970. Splanchnic metabolism of free fatty acids and production of triglyceride of very low density lipoproteins in normotriglyceridemic and hypertriglyceridemic humans. J. Clin. Invest. 49:2017-2035.

51. Kral, J.G., Lundholm, K., Bjorntorp, P., Sjostrom, L., and Schersten, T. 1977. Hepatic lipid metabolism in severe human obesity. Metabolism. 26:1025-1031.

52. Zelman, S. 1952. The liver in obesity. Arch. Intern. Med. 90:141-156.

53. Bjorntorp, P. 1988. Are regional metabolic differences of adipose tissue responsible for different risks of obesity? Horm. Metab. Res. Suppl. 19:23-25.

54. Micheli, H., Carlson, L.A., and Hallberg, D. 1969. Comparison of lipolysis in human subcutaneous and omental adipose tissue with regard to effects of noradrenaline, theophylline, prostaglandin E and age. Acta Chir. Scand. 135:663-670

55. Fujioka, S., Matsuzawa, Y., Tokunaga, K., and Tarui, S. 1987. Contributions of intra-abdominal fat accumulation to the impairment of glucose and lipid metabolism in human obesity. Metabolism. 36:54-59.

56. Egusa, G., Beltz, W.F., Grundy, S.M., and Howard, B.V. 1985. Influence of obesity on the metabolism of apolipoprotein B in humans. J. Clin. Invest. 76:596-603

57. Kissebah, A.H., Alfarsi, S., and Adams, P.W. 1981. Integrated regulation of very low density lipoprotein triglyceride and apolipoprotein-B kinetics in man: normolipidemic subjects, familial hypertriglyceridemia and familial combined hyperlipidemia. Metabolism. 30:856-868.

58. Roust, L.R., Kottke, B.A., and Jensen, M.D. 1994. Serum lipid responses to a eucaloric high-complex carbohydrate diet in different obesity phenotypes. Mayo Clin. Proc. 69:930-936.

59. Windler, E., et al. 1996. Differences in the mechanisms of uptake and endocytosis of small and large chylomicron remnants by rat liver. Hepatology. 24:344-351.

60. Hultin, M., Savonen, R., and Olivecrona, T. 1996. Chylomicron metabolism in rats: lipolysis, recirculation of core lipids as analyzed by compartmental modelling. J. Lipid Res. 37:1022-1036.

61. Karpe, F., Olivecrona, T., Hamsten, A., and Hultin, M. 1997. Chylomicron/chylomicron remnant turnover in humans: evidence for margination of chylomicrons and poor conversion of larger to smaller chylomicron remnants. J. Lipid Res. 38:949-961.

62. Miller, M. 1998. Is hypertriglyceridaemia an independent risk factor for coronary heart disease? Eur. Heart J. 19(Suppl. H):H18-H22. 